\section{耍 Heighten Science P U B L I C I T I O N S Corporation ISSN 2575-0143}

\title{
Concentration Polarization of Ox-LDL and Its Effect on Cell Proliferation and Apoptosis in Human Endothelial Cells
}

\author{
Shijie Liu ${ }^{1}$, Jawahar L Mehta ${ }^{1}$, Yubo Fan², Xiaoyan Deng ${ }^{2}$ and \\ Zufeng Ding ${ }^{1,2 *}$ \\ 'Department of Medicine, Central Arkansas Veterans Healthcare System and the University of \\ Arkansas for Medical Sciences, Little Rock, Arkansas, USA \\ ${ }^{2}$ Key Laboratory for Biomechanics and Mechanobiology of Ministry of Education, School of \\ Biological Science and Medical Engineering, Beihang University, Beijing 100191, China
}

\begin{abstract}
*Address for Correspondence: Dr. Zufeng Ding, Division of Cardiovascular Medicine, University of Arkansas for Medical Sciences, Little Rock, AR 72212, USA, Tel: 501-257-5558; Email: ZDing@uams.edu

Submitted: 14 December 2016 Approved: 29 December 2016 Published: 30 December 2016

Copyright: @ 2016 Ding Z, et al. This is an open access article distributed under the Creative Commons Attribution License, which permits unrestricted use, distribution, and reproduction in any medium, provided the original work is properly cited.
\end{abstract}

Keywords: Apoptosis; Atherosclerosis; Concentration polarization; LOX-1; Ox-LDL

\section{ABSTRACT}

Background: Flow-dependent concentration polarization of native LDL is important in the localization of atherogenesis. However, ox-LDL plays a more important role than n-LDL in atherogenesis by inducing cell proliferation and apoptosis. We hypothesized that concentration polarization of ox-LDL may adversely affect vascular beds due to its toxicity to endothelial cell (EC) lining.

Methods: Using a parallel-plate flow chamber technique, we studied water filtration rate and wall concentration of ox-LDLs EC monolayers cultured on permeable or non-permeable membranes. ECs cultured on permeable and non-permeable membranes were examined in terms of cell viability, ox-LDL uptake, LOX-1 expression and cell apoptosis (Cytochrome $\mathrm{c}$ and $\mathrm{Bcl}-2$ expression). We observed that the wall concentration of ox-LDL was about $16 \%$ higher in the permeable group than in the permeable group $(P<0.05)$. Cell proliferation (MTT assay) increased in response to low concentration of ox-LDL $(1-5 \mu \mathrm{g} / \mathrm{ml})$, and fell drastically in response to higher concentration; all these changes were more pronounced in the permeable group than in the nonpermeable group. The uptake of ox-LDL and LOX-1 expression by ECs were also significantly higher in the permeable group than in the non-permeable group of cultured cells.

Conclusion: These observations suggest that concentration polarization of ox-LDL occurs in an artery that is permeable to water, and ox-LDL concentration polarization can enhance ox-LDL accumulation into the arterial wall and accelerate EC proliferation at low concentrations and apoptosis at high concentrations, possibly via LOX-1 expression.

\section{INTRODUCTION}

There is increasing evidence to suggest that oxidative modification of low density lipoproteins (ox-LDL) in the arterial wall plays a crucial role in the initiation and progression of atherogenesis [1,2]. Lectin-like oxidized low-density lipoprotein scavenger receptor-1 (LOX-1) is one of the major receptors responsible for binding, internalizing and degrading ox-LDL, especially in endothelial cells (ECs) [3]. Activation of LOX-1 has been known to be related to many pathophysiological events, including EC and vascular smooth muscle cell (VSMC) proliferation, alteration in cell cycle signals and apoptosis [3,4]. Moreover, EC lining the blood vessel wall becomes more permeable to atherogenic lipids when subjected to ox-LDL [5]. This process leads to the accumulation of atherogenic lipids in the arterial wall, allowing formation of foam cells. 
Ox-LDL, particularly in large concentration, is cytotoxic and induces both apoptosis and necrosis in cultured ECs and VSMCs [6,7]. Atherosclerosis is associated with an increase in EC turnover, suggesting that EC apoptosis plays a fundamental role in the pathology of atherosclerosis [8]. Ross [9] found that the morphological changes in cultured ECs associated with ox-LDL toxicity are similar to those in the ECs covering atherosclerotic areas. Others $[10,11]$ found that ox-LDL can induce EC contraction and formation of intercellular gaps. On the other hand, ox-LDL in low concentration can induce EC proliferation [12]. Vascular proliferation and inflammation have been shown to be linked to the development vascular occlusive disease [13].

It has widely recognized that flow-induced shear stress is one of the most important hemodynamic factors in the localization of atherogenesis [14]. Accumulation of cholesterol and other lipids within the arterial wall is a hall-mark of atherogenesis; as such many groups of investigators are looking at the phenomenon of mass transport in circulation and the interactions of blood cells and lipids with the blood vessel walls [15]. From the viewpoint of mass transport, 'residence time' of atherogenic particles [16] and their deposition into the blood vessel walls [17] could account for the localization of atherogenesis in certain areas.

Deng et al. [18] have predicted a mass transport phenomenon of concentration polarization of atherogenic LDLs and verified it experimentally in vitro [19]. The phenomenon of LDL concentration polarization could be the basis of 'residence time' and the deposition of atherogenic particles. They suggested that flow-dependent LDL concentration at the blood/wall interface may play an important role in the localization of atherogenesis.

Since ox-LDL is more important than native LDL in atherogenesis, we hypothesized that concentration polarization of ox-LDL, if present, may result in increased EC apoptosis. This would result in enhanced EC turnover and occurrence of intercellular gaps facilitating the rate of ox-LDL infiltration/accumulation within the arterial wall. To verify this hypothesis, we used a parallel-plate flow chamber technique with human ECs cultured on permeable or non-permeable membranes and calculated water filtration rate and wall concentration of ox-LDL. We also studied EC viability and apoptosis as well as ox-LDL uptake.

\section{MATERIALS AND METHODS}

\section{Lipoprotein isolation, modification, and labeling}

Native LDL was prepared and purified from fresh plasma obtained from healthy volunteers by gradient ultracentrifugation [20]. Ox-LDL was prepared as described by Cominacini et al. [21]. DiI-ox-LDL was prepared by addition of $75 \mu \mathrm{l} \mathrm{of} 3 \mathrm{mg} / \mathrm{ml}$ 1,1'-dioctadecyl-3,3,3',' '-tetramethyl indocarbocyanine (DiI, Biomedical Technoligies Inc) to ox-LDL in $8 \mathrm{ml}$ of lipoprotein-deficient serum ( $\mathrm{d}>1.21 \mathrm{~g} / \mathrm{ml}$ plasma fraction). After overnight incubation at $37^{\circ} \mathrm{C}$, labeled lipoproteins were re-isolated by ultracentrifugation.

\section{Human arterial ECs monolayer cultures}

A suspension of $6-10^{\text {th }}$ generation ECs containing $5 \times 10^{6}$ cells in $0.5 \mathrm{ml}$ of the medium used for cell culture were seeded on: i) a glass slide covered with a membrane nonpermeable to plasma (Non-permeable group); or, ii) a glass slide covered with MillicellCM membrane (PICM 03050; Millipore Corp., Bedford, MA) which was permeable to plasma with pores of $0.4 \mu \mathrm{m}$ in diameter. The cells were then incubated for 3 hours at $37^{\circ} \mathrm{C}$. Then the cell culture was immersed in a culture medium. The state of attachment of cells onto the membrane and confluence of cells on the membranes were monitored by a phase contrast microscope from time to time. 


\section{Experimental setup}

Figure 1A shows a schematic drawing of the perfusion system. A pressure transducer and a flow meter were used to monitor the perfusion pressure and flow rate through the flow chamber, respectively. During the experiment, the flow chamber was enclosed in a container to keep it at a constant temperature of $37^{\circ} \mathrm{C}$.

Wall shear stress was calculated using the formula: $\tau=6 \mu \mathrm{Q} / \mathrm{wh}^{2}$, where $\mu$ is the viscosity of the medium and $\mathrm{Q}$ is the flow rate, $\mathrm{h}$ and $\mathrm{w}$ are the width and height of the parallel-plate flow chamber.

During the measurements, steady wall shear stress and perfusion pressure in the flow chamber were kept at $1.3 \mathrm{~Pa}$ and $100 \mathrm{mmHg}$, respectively, while the ox-LDL concentration in the perfusate was varied between 0 to $200 \mu \mathrm{g} / \mathrm{ml}$. The flow was maintained for $24 \mathrm{~h}$ except for Dil-ox-LDL uptake when the flow was for $2 \mathrm{~h}$.

\section{Filtration rate measurement}

For each experiment, the filtration rate across the wall of the cell culture insert was measured following the same procedure described by Deng et al. [18] with the help of the calibrated pipette on the dish support, which had an inner diameter of $1 \mathrm{~mm}$ (Figure 1A).

\section{Measurement of Dil-ox-LDL wall concentration}

The concentration of DiI-ox-LDL at the luminal surface of ECs layer was assessed by measuring the fluorescence intensity of the DiI-ox-LDL with a confocal laser microscope (SPII, Leica, Heidelberg, Germany). Briefly, after 24 hour of circulation, the field of vision of the microscope was adjusted to the central region of the cell culture and the

A.

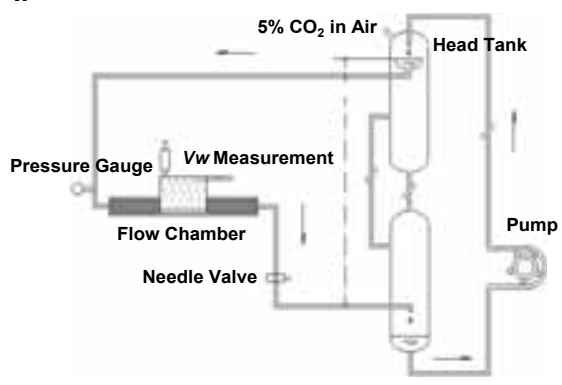

B.

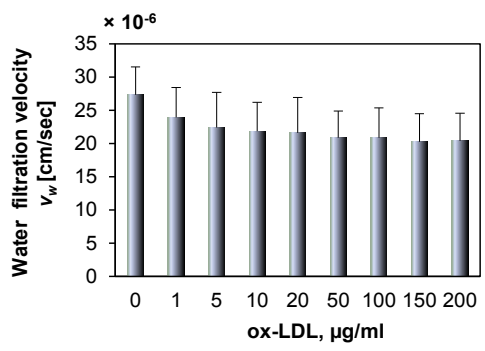

C.

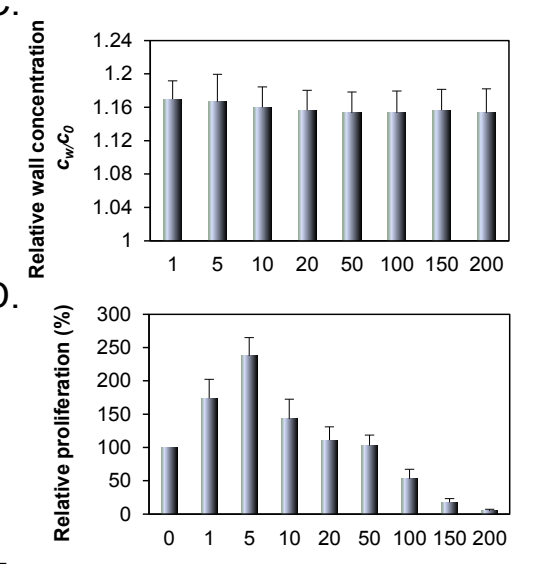

E.

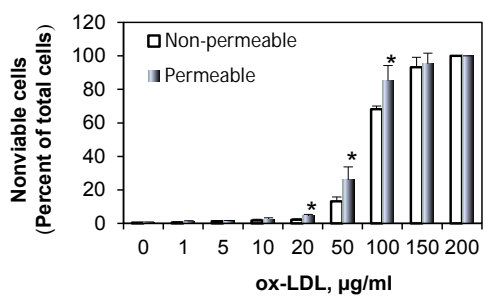

Figure 1: A. Schematic drawing of the perfusion system. The overflow head-tank provided a steady flow to the parallel-plate flow chamber. The filtration rate measurement cell on the abluminal side of the culture was filled with the same fluid as the perfusate. B. Water filtration rate for the permeable group with concentration of Dilox-LDL varied from 1 to $200 \mu \mathrm{g} / \mathrm{ml}$. C. Wall concentration of Dil-ox-LDL in permeable group was assessed by measuring the fluorescence intensity of Dil-ox-LDL using a confocal laser microscope. All data were normalized with the bulk concentration, $\mathrm{CO}$. D. Effect of ox-LDL on EC proliferation in endothelial cells was assessed by MTT assay. E. Trypan blue staining data in ECs cultured on permeable or non-permeable membranes and treated with ox-LDL ( 1 to $200 \mu \mathrm{g} / \mathrm{ml}$ ). EC suspensions were exposed to perfusion pressure of $100 \mathrm{mmHg}$ for $24 \mathrm{~h}$. The results are expressed as means $\pm S D(n=6)$. ${ }^{*}<0.05$ between permeable and non-permeable groups. 
focus adjusted to the surface of the cell culture using the phase-contrast mode. The intensities were standardized against the values obtained in the perfusion solution.

\section{Cell proliferation and viability assay}

Cell proliferation was determined by MTT assay and trypan blue exclusion (Invitrogen, Grand Island, NY) according to supplied protocols.

\section{Measurement of Dil-ox-LDL uptake}

Cultures endothelial cells were superfused with Dil-ox-LDL $5 \mu \mathrm{g} / \mathrm{ml}$ for $2 \mathrm{~h}$ at $37^{\circ} \mathrm{C}$. Then the cells were gently washed with PBS three times and digested with TrypsinEDTA. Dil-ox-LDL uptake was measured using flow cytometry (Becton Dickinson, Franklin Lakes, NJ) [22].

\section{Analysis of apoptosis}

Apoptosis was analyzed by Western blotting and Annexin-V/FITC kit (Bender MedSystems, Vienna, Austria) according to supplied protocols. Total Annexin-V activity was assessed by flow cytometry (Becton Dickinson, Franklin Lakes, NJ), and the results were analyzed with WinMDI29 software (Becton Dickinson).

\section{Statistical analysis}

Data from at least three sets of samples were used for statistical analysis. Results are shown as means $\pm \mathrm{SD}$. Multiple means were compared using a one-way analysis of variance (ANOVA). A student paired t-test was used to assess the significant differences between two groups. $\mathrm{P}<0.05$ was considered significant.

\section{RESULTS}

\section{Wall concentration of Dil-ox-LDLs and cell proliferation and death}

Figure 1B shows water filtration rate $\left(\mathrm{v}_{\mathrm{w}}\right)$ across the cell monolayer for the permeable group with various concentrations of DiI-ox-LDLs. As shown in the figure, $\mathrm{v}_{\mathrm{w}}$ was varied from 20.4 to $27.3 \times 10^{-6} \mathrm{~cm} / \mathrm{s}$. It was the highest when no DiIox-LDLs were added in the perfusion solution, and decreased slightly with increasing concentration of DiI-ox-LDLs in the perfusion solution. For the non-permeable group, the water filtration rate was zero (data not shown in the figure). Figure 1C shows wall concentration of DiI-ox-LDL $\left(\mathrm{c}_{\mathrm{w}}\right)$ in permeable group. As shown in the figure, the relative wall concentration, $\mathrm{c}_{\mathrm{w} /} \mathrm{c}_{0}$, is higher than 1.0 in the permeable group, indicating that concentration polarization occurred at the EC surface. The measurement showed that $\mathrm{c}_{\mathrm{w} /} \mathrm{c}_{0}$ remained almost unchanged with $\mathrm{c}_{0}$, and $\mathrm{c}_{\mathrm{w}}$ was about $16 \%$ higher than $\mathrm{c}_{0}$ in all conditions. Different from the permeable group, $\mathrm{c}_{\mathrm{w} /} \mathrm{c}_{0}$ was 1.0 for the non-permeable group (data not shown).

Figure 1D shows the effect of ox-LDL concentration (1 to $200 \mu \mathrm{g} / \mathrm{ml}$ ) on cell proliferation. Cell proliferation increased in response to low ox-LDL concentration, reaching its highest value at $5 \mu \mathrm{g} / \mathrm{ml}$, and then dropped drastically in response to higher concentrations, indicating that high concentrations of ox-LDL are toxic to ECs. To determine the cytotoxic concentration of ox-LDL, ECs cultured on the permeable or the non-permeable membranes were exposed to ox-LDL suspensions of different concentrations for $24 \mathrm{~h}$. Trypan blue staining revealed very few dead cells in the untreated control cells (Figure 1E). The treatment of ECs with ox-LDL suspension led to increasing proportion of nonviable cells in a dose-dependent fashion for both the permeable and the non-permeable groups. At low concentration (in the range of 1 to $20 \mu \mathrm{g} / \mathrm{ml}$ ), nonviable cells in both groups were similar; however, the proportion of nonviable cells increased very sharply with increasing concentration of ox-LDL (50 to $200 \mu \mathrm{g} / \mathrm{ml}$ ). These observations complement the data from MTT assay. 


\section{Dil-ox-LDL uptake and its effect of LOX-1 expression}

Figure 2A illustrates DiI-ox-LDL uptake by the ECs. As evident from the figure, DiIox-LDL uptake by ECs was correlated with the concentration of DiI-ox-LDL for both groups, but the correlation was highly non-linear. At low concentrations (1 to $10 \mu \mathrm{g} /$ $\mathrm{ml}$ ), DiI-ox-LDL uptake increased gradually. When the concentration of DiI-ox-LDLs increased from 20 to $100 \mu \mathrm{g} / \mathrm{ml}$, DiI-ox-LDL uptake increased very sharply. Beyond $150 \mu \mathrm{g} / \mathrm{ml}$, the uptake curve reached a plateau. Importantly, DiI-ox-LDL uptake in the permeable group were obviously higher than those in the non-permeable group.

Figure 2B shows the correlation between DiI-ox-LDL uptake by ECs and the concentration of ox-LDL on the luminal surface of the cell culture insert. As evident, DiI-ox-LDL uptake by positively correlated with the wall concentration of DiI-ox-LDL. However, the correlation was highly non-linear. When $\mathrm{c}_{\mathrm{w}}$ was low, the uptake increased very sharply; on the other hand, the increase in uptake increase became gradual with increasing $\mathrm{C}_{\mathrm{w}}$ and the reach a plateau beyond $\mathrm{C}_{\mathrm{w}}$ value of $115 \mu \mathrm{g} / \mathrm{ml}$.

In consonant with DiI-ox-LDL uptake, ox-LDL induced LOX-1 expression in a dosedependent manner in both groups. Due to concentration polarization of ox-LDL on the cell surface, LOX-1 expression was greater in the permeable group as compared with the non-permeable group at all concentrations.

\section{Concentration polarization of ox-LDL-induced apoptosis in ECs}

Figure 3A shows representative examples of ox-LDL induced apoptosis in ECs cultured on the permeable and the non-permeable membranes. As shown in the Figure 3A, the apoptosis increased with increasing concentration of ox-LDLs in both the permeable and non-permeable groups. But the apoptosis rate in the permeable group was always higher than that in the non-permeable group. It is well known that apoptosis is regulated by various pro-apoptosis-related proteins Cytochrome c, and anti-apoptosis-related proteins Bcl-2 [22]. As shown in Figure 3B and 3C, ox-LDL in a concentration-dependent manner induced the expression of Cytochrome c (Figure 3B) and inhibited the expression of Bcl-2 (Figure 3C), supporting the observations from flow cytometry. Further, apoptosis of ECs in response to ox-LDL was greater in permeable group compared to non-permeable group.

A.
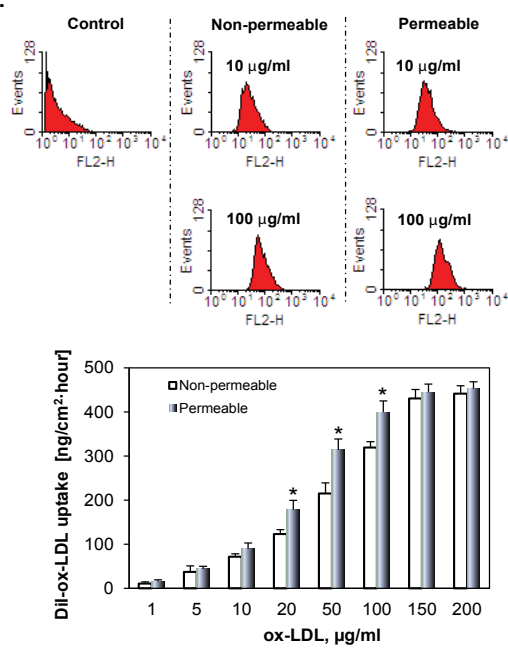

B.

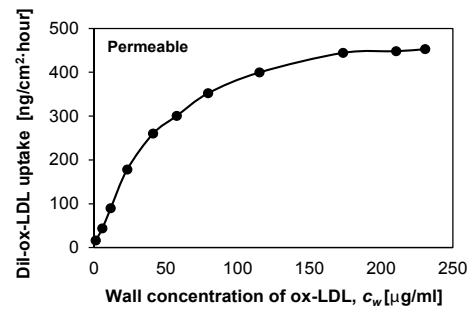

C.

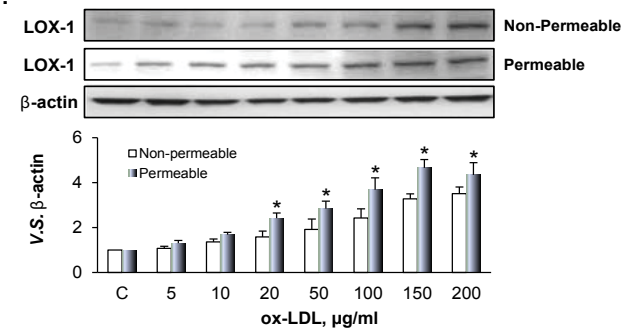

Figure 2: A. Dil-ox-LDL uptake by ECs cultured on permeable and non-permeable membranes and treated with varied ox-LDL suspensions for $24 \mathrm{~h}$. B. Effect of the wall concentration of Dil-LDL, Cw, on Dil-ox-LDL uptake. At low $\mathrm{C}$, ox-LDL uptake rose very sharply, thereafter the ox-LDL uptake became gradual. A plateau seemingly exists on the uptake. C. LOX-1 expression in permeable and non-permeable groups measured by western blot. The results are expressed as means $\pm S D(n=6)$. ${ }^{*}<0.05$ between permeable and non-permeable groups. 
A.

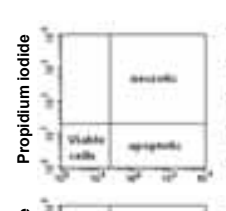

Non-permeable
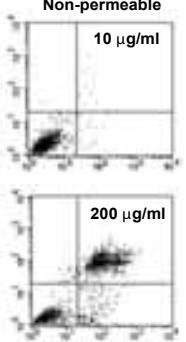

Annexin-V FITC

B.
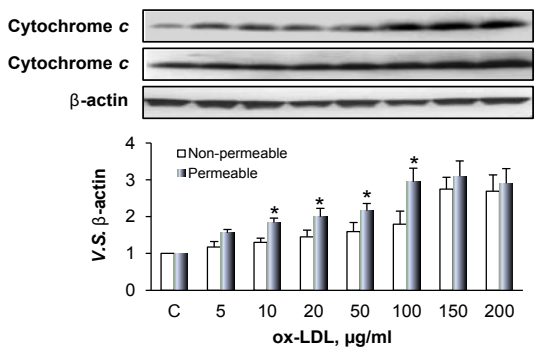

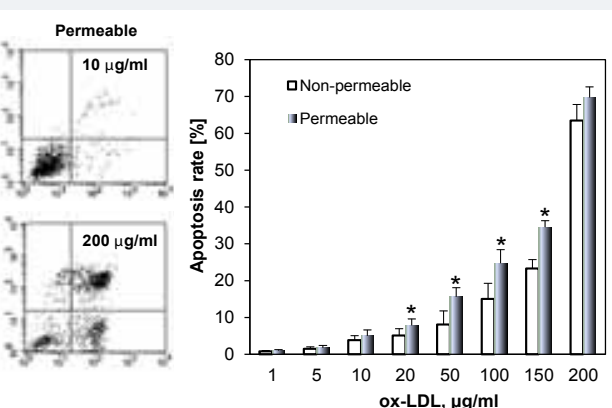

C.

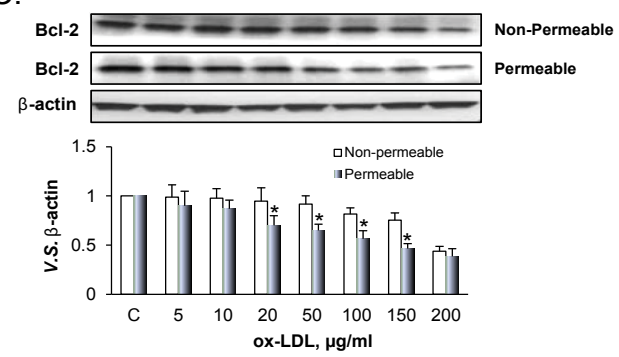

Figure 3: Death of ECs treated with different concentration of ox-LDL. A. Apoptotic and necrotic deaths in distinguished by using AV label and PI stain. The percentage of apoptotic cell population was determined by flow cytometry. B. Cytochrome $\mathrm{c}$ and $\mathrm{Bcl}-2$ expression by ECs cultured on the permeable and non-permeable membranes. The results are expressed as means $\pm S D(n=6) .{ }^{\star} P<0.05$ between permeable and non-permeable groups.

\section{DISCUSSION}

A typical feature of atherosclerotic plaques is the accumulation of ox-LDL within the arterial wall [9]. Ox-LDL contributes to the inflammatory state of atherosclerosis and plays a key role in atherogenesis [23]. Because ECs display low permeability to plasma proteins, it has been suggested that the flow across the artery wall may cause concentration polarization of LDL with the LDL increasing in concentration from bulk value towards interface within the arterial system [18]. The mass transport phenomenon of concentration polarization of LDL has been confirmed both theoretically and experimentally [19]. In the present study, we hypothesized that concentration polarization of ox-LDLs might occur leading to enhanced ox-LDL uptake by the arterial wall. This might in turn accelerate EC death and apoptosis. To substantiate this hypothesis, we studied water filtration rate and wall concentration of ox-LDL in an in vitro model wherein ECs were cultured on permeable and nonpermeable membranes.

In the present study, steady wall shear stress and perfusion pressure in the flow chamber were kept at $1.3 \mathrm{~Pa}$ and $100 \mathrm{mmHg}$ respectively, which are the average of physical levels. Our data demonstrated that the wall concentration of ox-LDL ( $\mathrm{c}_{\mathrm{w}}$ ) was about $16 \%$ higher in the permeable group related to increased filtration, indicating that concentration polarization of ox-LDL indeed occurred on the surface of the cultured ECs. In the non-permeable group, because water filtration rate across the cell monolayer was zero, the value of $c_{w}$ remained the same as that of $c_{0}$. Consequently, Dil-ox-LDL uptake by ECs was also higher for the permeable group than that in the non-permeable group. We believe this resulted in increased EC LOX-1 expression and apoptosis in the permeable group than in the non-permeable group.

Xavier et al. [23] found that ox-LDL inhibits proliferation and spontaneous motility of ECs, which positively correlated with the degree of oxidation of LDL and its concentration. High concentration of ox-LDL clearly has a cytotoxic effect on ECs [24], whereas at low concentrations, ox-LDL can induce EC proliferation. Both actions are 
mediated in large part by LOX-1 expression and activation [24]. In keeping with this concept, Galle et al. [12] showed a dual effect of ox-LDL on cell cycle in ECs. Several investigators $[10,11]$ have shown that ox-LDL induces EC contraction and intercellular gaps, which would increase filtration flow across the arterial wall. Accumulation of oxLDL in the subendothelial layers would then cause lysis of cellular components, such as VSMCs and macrophages, a phenomenon that could be responsible for the formation of the necrotic core, a characteristic of advanced atherosclerotic lesion [25].

Using a complementary DNA expression library derived from bovine aortic ECs, Sawamura et al [26] first discovered that LOX-1 is a major receptor for ox-LDL in ECs $[27,28]$. The present study revealed that DiI-ox-LDL uptake by ECs was positively but non-linearly correlated with the concentration of ox-LDL. The measured Dil-ox-LDL uptake possibly consisted of 2 parts: the first part reflected LOX-1-mediated bindings of ox-LDL, and the second part reflected ox-LDL infiltration/accumulation. At very high concentration the toxicity of ox-LDL led to a very large number of nonviable cells and high apoptosis rate. Therefore, ox-LDL uptake by the cells plateaued or even fell.

While studying the effect of ox-LDL on ox-LDL uptake and EC apoptosis, we noted significant differences between permeable and non-permeable groups only when the concentration of ox-LDL was $20-100 \mu \mathrm{g} / \mathrm{ml}$. This observation suggests that concentration polarization of ox-LDL is biologically relevant only in a range of ox-LDL concentration.

\section{CONCLUSION}

The present study provides evidence to support that concentration polarization of ox-LDL indeed occurs at intima of the artery that is permeable to water, and the occurrence of ox-LDL concentration polarization regulates ox-LDL accumulation and cell apoptosis.

\section{ACKNOWLEDGMENTS}

This study was supported by funds from the Department of Veterans Affairs, Veterans Health Administration, Office of Research and Development, Biomedical Laboratory Research and Development, Washington, DC. Additional support was provided by Grants-in-Aid from the National Natural Science Foundation of China (No. 11332003, 11572028).

\section{REFERENCES}

1. Chistiakov DA, Melnichenko A A, Orekhov AN, Bobryshev $\mathrm{Y}$ V. Paraoxonase and atherosclerosis-related cardiovascular diseases. Biochimie. 2017; 132: 19-27. Ref.: https://goo.gl/Y1CLFa

2. Mehta JL, Chen J, Hermonat PL, Romeo F, Novelli G. Lectin-like oxidized low-density lipoprotein receptor-1 (LOX-1): a critical player in the development of atherosclerosis and related disorders. Cardiovas Res. 2006; 69: 36-45. Ref.: https://goo.gl/uZOz8t

3. Ding Z, Liu S, Wang X, Mathur P, Dai Y, et al. Cross-Talk between PCSK9 and Damaged mtDNA in Vascular Smooth Muscle Cells: Role in Apoptosis. Antioxid Redox Signal. 2016; 25 997-1008. Ref.: https://goo.gl/zjwncZ

4. Ding Z, Liu S, Wang X, Deng X, Fan Y, et al. Cross-talk between LOX-1 and PCSK9 in vascular tissues. Cardiovasc Res. 2015; 107: 556-567. Ref.: https://goo.gl/jzNZVd

5. Chistiakov DA, Orekhov AN, Bobryshev YV. LOX-1-Mediated Effects on Vascular Cells in Atherosclerosis. Cell Physiol Biochem. 2016; 38: 1851-1859. Ref.: https://goo.gl/hukwv1

6. Cobbold CA, Sherratt JA, Maxwell SR. Lipoprotein oxidation and its significance for atherosclerosis: a mathematical approach. Bull Math Biol. 2002; 64: 65-95. Ref.: https://goo.gl/PTDi3B

7. Chen XP, Xun KL, Wu Q, Zhang TT, Shi JS, et al. Oxidized low density lipoprotein receptor-1 mediates oxidized low density lipoprotein-induced apoptosis in human umbilical vein endothelial cells: Role of reactive oxygen species. Vasc Pharmacol. 2007; 47: 1-9. Ref.: https://goo.gl/o77VPB

8. Dimmeler S, Zeiher AM. Endothelial cell apoptosis in angiogenesis and vessel regression. Circ Res. 2000; 87: 434439. Ref.: https://goo.gl/60sOkr 
9. Ross R. The pathogenesis of atherosclerosis: a perspective for the 1990s. Nature. 1993; 362: 801-809. Ref.: https://goo.gl/MDN1Xk

10. Essler M, Retzer M, Bauer M, Heemskerk JW, Aepfelbacher M, et al. Mildly oxidized low density lipoprotein induces contraction of human endothelial cells through activation of Rho/Rho kinase and inhibition of myosin light chain phosphatase. J Biol Chem. 1999; 274: 30361-30364. Ref.: https://goo.gl/rc2x0v

11. Zhao B, Ehringer WD, Dierichs R, Miller FN. Oxidized low-density lipoprotein increases endothelial intracellular calcium and alters cytoskeletal F-actin distribution. Eur J Clin Invest. 1997; 27: 48-54. Ref.: https://goo.gl/4Q0JSw

12. Galle J, Heinloth A, Wanner C, Heermeier K. Dual effect of oxidized LDL on cell cycle in human endothelial cells through oxidative stress. Kidney Int Suppl. 2001; 78: 120-123. Ref.: https://goo.gl/zic9cl

13. Dzau VJ, Braun-Dullaeus RC, Sedding DG. Vascular proliferation and atherosclerosis: New perspectives and therapeutic strategies. Nat Med. 2002; 8: 1249-1256. Ref.: https://goo.gl/0e4mzG

14. Ding Z, Liu S, Deng X, Fan Y, Wang X, et al. Hemodynamic shear stress modulates endothelial cell autophagy: Role of LOX-1. Int J Cardiol. 2015; 1: 86-95. Ref.: https://goo.gl/PJUlaF

15. Kleinstreuer C, Hyun S, Buchanan JR Jr, Longest PW, Archie JP Jr, et al. Hemodynamic parameters and early intimal thickening in branching blood vessels. Crit Rev Biomed Eng. 2001; 29: 1-64. Ref.: https://goo.gl/tT2605

16. Ku DN, Giddens DP. Pulsatile flow in a model carotid bifurcation. Arteriosclerosis. 1983; 3: 31-39. Ref.: https://goo.gl/HGPmp7

17. Hyun S, Kleinstreuer C, Archie JP Jr. Computational particle-hemodynamics analysis and geometric reconstruction after carotid endarterectomy. Comput Biol Med. 2001; 31: 365-384. Ref.: https://goo.gl/1tePMz

18. Deng $X$, Marois $Y$, How T, Merhi Y, King M, et al. Luminal surface concentration of lipoprotein (LDL) and its effect on the wall uptake of cholesterol by canine carotid arteries. J Vasc Surg. 1995; 21: 135-145. Ref.: https://goo.gl/a00uVh

19. Ding Z, Liu S, Wang X, Deng X, Fan Y, et al. Hemodynamic shear stress via ROS modulates PCSK9 expression in human vascular endothelial and smooth muscle cells and along the mouse aorta. Antioxid Redox Signal. 2015 22: 760-771. Ref.: https://goo.gl/bbl3e3

20. Hafiane A, Genest J. High density lipoproteins: Measurement techniques and potential biomarkers of cardiovascular risk. BBA Clin. 2015; 31: 175-188. Ref.: https://goo.gl/eX587z

21. Cominacini L, Garbin U, Davoli A, Micciolo R, Bosello O, et al. A simple test for predisposition to LDL oxidation based on the fluorescence development during copper-catalyzed oxidative modification. J Lipid Res. 1991; 32 349-358. Ref.: https://goo.gl/VawjTD

22. Ding Z, Liu S, Wang X, Dai Y, Khaidakov M, et al. LOX-1, mtDNA damage, and NLRP3 inflammasome activation in macrophages: implications in atherogenesis. Cardiovasc Res. 2014; 103: 619-628. Ref.: https://goo.gl/AudeOr

23. Xavier HT, Abdalla DS, Martinez TL, Ramires JA, Gagliardi AR. Effects of oxidized LDL on in vitro proliferation and spontaneous motility of human coronary artery endothelial cells. Arq Bras Cardiol. 2004; 83: 493-497. Ref.: https://goo.gl/jbzG1a

24. Dandapat A, Hu C, Sun L, Mehta JL. Small concentrations of oxLDL induce capillary tube formation from endothelial cells via LOX-1-dependent redox-sensitive pathway. Arterioscler Thromb Vasc Biol. 2007; 27: 24352442. Ref.: https://goo.gl/bRtQ65

25. Hoff HF, O'Neil JA. Oxidation of LDL: role in atherogenesis. Klin Wochenschf. 1991; 69: 1032-1038. Ref. https://goo.gl/QbT220

26. Sawamura T, Kume N, Aoyama T, Moriwaki H, Hoshikawa $\mathrm{H}$, et al. An endothelial receptor for oxidized low-density lipoprotein. Nature. 1997; 386: 73-77. Ref.: https://goo.gl/WY4hsp

27. Ding Z, Liu S, Wang $X$, Khaidakov M, Dai $Y$, et al. Oxidant stress in mitochondrial DNA damage, autophagy and inflammation in atherosclerosis. Sci Rep. 2013; 3: 1077. Ref.: https://goo.gl/mzir86

28. Moriwaki H, Kume N, Sawamura T, Aoyama T, Hoshikawa H, et al. Ligand specificity of LOX-1, a novel endothelial receptor for oxidized low density lipoprotein. Arterioscler Thromb Vasc Biol. 1998; 18: 1541-1547. Ref.: https://goo.gl/37cwpW 